Original

\title{
El aprendizaje de la Prostatectomía Radical Laparoscópica según el modelo de Leipzig. Análisis de un programa tutelado progresivo
}

\author{
Miguel Ramírez Backhaus, Jens Uwe Stolzenburg*, Mihn Do*, Ania Dietel*, José L. Ruiz-Cerdá, \\ Juan F. Jiménez Cruz
}

Servicio de Urología. Hospital Universitario La Fe. Valencia. España. *Departamento de Urología. Uniklinik Leipzig. Alemania.

\begin{abstract}
Resumen
Introducción y objetivo: En la mayoría de los casos el aprendizaje de la prostatectomía radical laparoscópica no se completa durante la residencia. Ante esta problemática internacional surgen las becas o "fellowships" de los centros de excelencia europeos y norteamericanos. El objetivo del presente trabajo es evaluar la eficiencia de la beca de formación propuesta por el grupo alemán.

Material y método: Describimos la evolución de un becario durante el entrenamiento progresivo, tutorizado por módulos descrito por el grupo de Leipzig, evaluamos la eficiencia del modelo describiendo los resultados de las primeras 30 prostatectomías radicales del becario como cirujano autónomo, comparamos los resultados con la serie histórica del centro, con la curva de aprendizaje de residentes del mismo centro y de otros centros.

Resultados: El becario cumplimentó el entrenamiento por módulos en 7 meses posteriormente se le asignaron 30 prostatectomías radicales sin la obligatoriedad de ser ayudado por un cirujano experto. En el 46,67\% de los pacientes se aplicó una técnica intrafascial. El tiempo quirúrgico medio fue de $2 \mathrm{~h}$ y 27 minutos, incluida la linfadenectomía (realizada en 20/30 pacientes). Dos pacientes presentaron márgenes positivos, todas las anastomosis, menos un paciente, fueron estancas. La sonda se retiró al quinto día (mediana). No se detectaron complicaciones intraoperatorias ni en el posoperatorio tardío. Los resultados de éstas, se superponen a la de los residentes del mismo centro y a la de otros urólogos que siguen un entrenamiento tutelado de larga duración.

Conclusiones: El aprendizaje de la prostatectomía radical laparoscópica debe seguir un modelo tutorizado progresivo de larga duración. Hasta la expansión definitiva de la técnica, el programa de formación tipo "fellow" que imparte el grupo de Leipzig es un modelo de formación completo y efectivo.
\end{abstract}

Palabras clave: Cáncer de próstata. Prostatectomía radical. Curva de aprendizaje.

\section{Learning laparoscopic radical prostatectomy with the Leipzig program. Analysis of the training module program}

\section{Abstract}

Introduction and objective: In most cases the learning curve of laparoscopic radical prostatectomy is not completed during the residency. This is why International Fellowship programs are becoming more popular. The aim of this paper is to evaluate the efficiency of the fellowship program of the German group.

Material and methods: We describe the evolution of our last fellow in the modular training program developed by the Leipzig group. We also evaluate the efficiency of this program by analyzing the results of 30 procedures performed by the fellow as first surgeon. In addition we have reviewed the literature and compared those outcomes with previous series of the clinic and other training programs.

Results: The training programme was completed in four months. After this time, the fellow performed 30 procedures without any assistance from a tutor. $46,67 \%$ of these procedures were nerve sparing. The time average was 2 hours and 27 minutes. The programme includes lymphadenectomy in 20 patients. Two patients had positive margins, all anastomosis were tight except for one. Urethral catheter was removed on the fifth day and there were no other intraopertive or early postoperative complications. Results may be compared to previous results of other residents of the department and also of other groups where a long term tutor program was performed.

Conclusion: The learning process of laparoscopic radical prostatectomy should follow a long term tutorized model. Until the definitive expansion/development of this technique, the Leipzig Fellowship program is a complete and effective formation schedule.

Keywords: Prostate cancer. Radical prostatectomy. Learning curve. 
A sistimos a un crecimiento exponencial en el número de intervenciones laparoscópicas. Paralelamente, aumenta el número de cirujanos urólogos laparoscopistas en nuestro medio. Sin embargo, la formación laparoscópica de los residentes es, en líneas generales, incompleta.

El elevado interés que estas técnicas despierta entre los urólogos jóvenes se ve frenado por diversas circunstancias:

- La curva de aprendizaje es larga y tediosa, los resultados oncológicos y funcionales han de ser, al menos, tan buenos como las técnicas abiertas clásicas que la preceden.

- La casuística de la mayoría de los centros no terciarios impide la formación rápida o paralela de varios residentes y especialistas.

- La cirugía laparoscópica urológica carece de procedimientos sencillos que faciliten la incorporación progresiva a las modalidades mínimamente invasivas.

- La incorporación de los residentes supone un incremento de los tiempos quirúrgicos con el consiguiente costo económico.

La técnica laparoscópica más demandada, precisamente por su frecuencia, es la prostatectomía radical laparoscópica.

Hasta la fecha, con los datos que disponemos, en nuestro medio, sólo el Hospital La Paz forma desde el año 2006 a los residente de último año en esta técnica quirúrgica ${ }^{1}$.

Tanto en el medio anglosajón como en el norteamericano, se dan circunstancias parecidas. Consecuencia de dicha situación, en la última década, los centros de excelencia con mayor casuística ofrecen a los especialistas más jóvenes, "fellowship" o becas para la formación en cirugía laparoscópica, periodos de entrenamiento intensivo en esta $u$ otra modalidad quirúrgica. De hecho, la Sociedad Norteamericana de Endourología ofrece actualmente 53 "fellowships", la mayoría de ellos de cirugía laparoscópica.

En el presente trabajo describimos el programa de formación del Centro Internacional de Entrenamiento Laparoscópico de la Clínica Universitaria de Leipzig, la curva de aprendizaje y evolución del último becario "fellow", así como un estudio retrospectivo comparativo de los resultados con la series histórica del centro y con dos de los residentes que realizaron el mismo programa de entrenamiento ${ }^{2}$. En esta primera fase se tratará de demostrar la bondad del método de entrenamiento mediante la descripción de los resultados preliminares de los becarios y la reproducibilidad de la técnica descrita por el grupo.

\section{MATERIAL Y MÉTODO}

El Centro Internacional de Formación laparoscópica ofrece dos becas no retributivas de formación en prostatectomía radical endoscópica extraperitoneal (endoscopic extraperitoneal radical prostatectomy EERPE).

Beca o "fellowship" tiempo completo (6-8 meses).

Tutorias formativas intermitentes (1-6 semanas) ${ }^{3}$.

Ambos tratan de enseñar la EERPE mediante el programa de entrenamiento por módulos tutelado. La beca a tiempo completo está dirigida a especialistas jóvenes sin ser necesaria la experiencia laparoscópica previa. La segunda modalidad es una colaboración entre centros hospitalarios y acceden a ella especialistas de centros con menor casuística con experiencia previa y contempla el desplazamiento periódico de un tutor a dicho centro.

Los alumnos disponen de material multimedia, acceso a un laboratorio con tres "pelvic trainers" con sendas torres laparoscópicas con ópticas de alta definición, donde deberán entrenar los primeros meses la sutura endoscópica en un modelo de látex y con la distribución de los trocares descrita por el grupo ${ }^{4}$.

Durante la beca a tiempo completo el alumno cumplimenta el programa de entrenamiento por módulos descrito previamente por el grupo de Leipzig ${ }^{2}$ Tabla 1. Básicamente consiste en la estratificación de la cirugía en 5 módulos de dificultad creciente, el alumno realiza durante las cirugías el módulo correspondiente a su nivel, mientras que el resto son realizados por su tutor experto, con ello persiguen un aprendizaje progresivo sin interferir en los resultados y en los tiempos quirúrgicos. El paso de un nivel al inmediato superior es decidido por el tutor y no existe un número de repeticiones determinado. Dicho periodo, de duración variable, se continúa con cirugías en las que el becario, en función de la situación contextual de la enfermedad del paciente y de la dinámica de la jornada laboral realiza varios módulos, incluso la prostatectomía propiamente dicha, o bien la anastomosis; siendo el resto de los pasos cumplimentados por el tutor experto que previamente ejercía de primer ayudante. Finalmente el alumno es considerado apto para rea- 
Tabla 1

\begin{tabular}{llc}
\hline Orden & Descripción del paso quirúrgico & Módulo \\
\hline 1 & $\begin{array}{l}\text { Colocación de los trocares y } \\
\text { disección del espacio preperitoneal. }\end{array}$ & I \\
2 & Linfadenectomía pélvica & II \\
3 & $\begin{array}{l}\text { Incisión de la fascia endopélvica y } \\
\text { resección de los ligamentos } \\
\text { puboprostáticos }\end{array}$ & \\
& $\begin{array}{l}\text { Ligadura del complejo venosos dorsal } \\
4\end{array}$ & III \\
5 & $\begin{array}{l}\text { Disección dorsal y lateral del cuello } \\
\text { vesical }\end{array}$ & II \\
6 & Disección posterior del cuello vesical & III \\
7 & $\begin{array}{l}\text { Disección y resección de los } \\
\text { conductos deferentes }\end{array}$ & III \\
8 & Incisión de la fascia de Denonvilliers & III \\
9 & $\begin{array}{l}\text { Disección de los pedículos } \\
\text { vasculonerviosos }\end{array}$ & III \\
10 & Cirugía conservadora de bandeletas & V \\
11 & Disección apical & IV \\
12 & Anastomosis & III \\
& Circunferencia dorsal $4,5,6,7,8$ horas) & IV \\
& $\begin{array}{l}\text { Suturas de las } 3 \text { y } 9 \text { horas } \\
\text { Cierre del cuello vesical, si procede }\end{array}$ & y puntos de las 11 y 13 horas. \\
\hline
\end{tabular}

lizar el procedimiento por sí sólo y se le asignan un número variable de intervenciones en las que ejercerá como primer cirujano sin ser imprescindible la asistencia de un ayudante experto.

Se excluyen de la formación pacientes con contrato privado y pacientes con antecedentes de resección transuretral de la próstata, adenomectomía retropúbica, uretrotomía interna y prostatectomías de rescate tras HIFU $^{5}$ o braquiterapia. Dichos pacientes son intervenidos íntegramente por un cirujano experto.

La docencia es impartida por cuatro urólogos expertos en cirugía laparoscópica (JUS, MD, RR, $\mathrm{AD}$ ). El director del centro (JUS) decide cuándo el alumno puede ascender de nivel.

Evaluación del modelo mediante: descripción de la evolución progresiva de un alumno en las diferentes etapas formativas. Se describen las características clínicas de los pacientes intervenidos, el tipo de intervención, el tiempo quirúrgico, los resultados histológicos, las complicaciones intraoperatorias y postoperatorias así como los resultados funcionales en el posoperatorio inmediato. Se analizan las últi- mas prostatectomías radicales cuando el alumno ha alcanzado el último periodo del curso y se comparan de manera retrospectiva, dichos datos, con la curva de aprendizaje previa de dos urólogos formados en dicho centro, así como con los datos de otros métodos de aprendizaje tutelados.

Los datos se recogen de manera prospectiva.

\section{RESULTADOS}

Durante los meses de formación se llevaron a cabo 250 prostatectomías radicales, de las cuáles 157 (63\%) fueron consideradas aptas para la docencia.

El alumno carecía de experiencia alguna en prostatectomía radical retropúbica o perineal, no había realizado cursos previos de entrenamiento laparoscópico, aunque sí entrenamiento individualizado con "pelvic trainer", donde realizó 1000 puntos sueltos endoscópicos durante los primeros dos meses, en el modelo de látex.

La evolución progresiva por los diferentes módulos está descrita en la Tabla 2.

Tabla 2. Evolución del entrenamiento por módulos

\begin{tabular}{lc}
\hline & EERPE consecutivas \\
\hline Cámara & 4 \\
Primer ayudante & 4 \\
Modulo 1 & 3 \\
Modulo 2 & 18 \\
Modulo 3 & 8 \\
Modulo 4 & 13 \\
Modulo 5 & 2 \\
\hline Total & 52 \\
\hline
\end{tabular}

La primera semana, con el objetivo de memorizar el procedimiento estandarizado ${ }^{6,7}$, el alumno ejerció de segundo ayudante llevando la cámara, posteriormente como primer ayudante hasta que comenzó el entrenamiento por módulos propiamente dicho. El módulo 1, preparación del espacio preperitoneal con el "trocar balón”, inserción de los trócares, incisión de la fascia endopélvica y de los ligamentos puboprostáticos, fue realizado en tres pacientes, progresando al siguiente peldaño. Ahora sin embargo, fueron necesarias 18 repeticiones del módulo 2 hasta alcanzar el nivel requerido para el siguiente módulo. El módulo 3 fue repetido en 8 ocasiones consecutivas, en dos de ellas el alumno fue sustituido como cirujano por la incisión intraprostática iatrógena en 
un lóbulo medio. El módulo 4 que incluía los puntos de la anastomosis dorsal así como la delicada disección apical fue repetido 13 veces. Tras dichas intervenciones el alumno continuó con las modificaciones de la técnica intrafascial ${ }^{4}$.

Tras este periodo, que ocupó un total de 11 semanas, el becario fue autorizado a realizar partes consecutivas de la intervención en función de la situación contextual del paciente y de la jornada laboral. Tras 4 meses y dos semanas, el periodo de formación estaba cumplimentado. Globalmente el alumno ejerció en 25 procedimientos como segundo ayudante y en 130 como primero. Realizando un total de 157 módulos de entrenamiento.

A continuación, al becario se le asignaron 30 prostatectomías radicales. Las características clínicas de los pacientes están representadas en la Tabla 3.

Tabla 3. Características de los pacientes intervenidos por el becario

\begin{tabular}{ll}
\hline Cirugía intrafascial /exéresis amplia. & $14 / 16$ \\
Corrección de hernias asociada & 5 \\
pT2/pT3 & $26 / 4$ \\
R1. n (\%) & $2(6,67 \%)$ \\
Linfadenectomía pélvica. n (\%) & $20(66,67 \%)$ \\
Número de Ganglios. media (rango) & $19(9-35)$ \\
Tiempo quirúrgico. media (rango) & $2: 27(1: 20-3: 10)$ \\
Fuga anastomosis cistografía control n (\%) & $1(3,33 \%)$ \\
\hline
\end{tabular}

En 14 de las intervenciones se aplicó una técnica intrafascial con el fin de conservar los pedículos neurovasculares, en el resto la resección fue más amplia. El 66,67\% de los pacientes se asoció una linfadenectomía pélvica que incluía tejido linfático de la fosa obturatriz, la vena y arteria iliaca externa hasta la bifurcación y/o cruce con el uréter. Con una resección una media de 19 ganglios por paciente.

El tiempo medio de la intervención fue de 2 horas y 27 minutos, incluimos en dicho valor una cistoscopia protocolaria previa a la intervención, la linfadenectomía en los casos que se realizó 20/30 así como la corrección de hernias inguinales 5/30. Si excluimos las intervenciones que asociaban linfadenectomía, la duración media fue de 1 hora 57 minutos.

No se objetivaron complicaciones intraoperatorias ni en el postoperatorio temprano.

A todos los pacientes se les realizó una cistografía con $200 \mathrm{ml}$ de contraste yodado entre el tercer y quinto día de la intervención, la anastomosis estaba estanca en todos, salvo en un paciente. El catéter se retiró entre el cuarto y séptimo días. Al octavo día postoperatorio se controlaba el número de compresas que el paciente utilizó en 24 horas, resultando una media de 4 compresas (1-8).

En el $80 \%$ de los casos la anatomía patológica fue de adenocarcinoma de próstata en estadio T2 mientras que el 20\% T3. Sólo dos pacientes presentaron márgenes positivos, ambos en el ápex (T2c y T3a).

Los resultados de las prostatectomías radicales del alumno formado son comparadas detalladamente a las de los anteriores grupos y a las propias de los primeros residentes del centro alemán en la Tabla 4.

\section{DISCUSIÓN}

La prostatectomía radical laparoscópica es un procedimiento difícil con una curva de aprendizaje larga y tediosa. El aprendizaje tutelado y estructurado parece ser la estrategia más eficiente para alcanzar el nivel deseable ${ }^{4,8}$.

Dicho modelo de enseñanza no es nuevo, Halsted fue el primero que lo introdujo en la docencia de la cirugía convencional ${ }^{9}$.

En la técnica que nos ocupa, es muy complejo evaluar los diferentes programas de formación disponibles en la literatura, por la ingente cantidad de variables que entran en juego.

La Asociación Española de Urología comenzó en el año 2006 un programa de formación en el cual 37 expertos laparoscopistas primero mostraban en sus centros la técnica y posteriormente se trasladaban a los centros donde asistían a los alumnos. 141 urólogos especialistas recibieron el entrenamiento en cirugía renal y pélvica. No disponemos de datos de eficiencia del programa, pero a nuestro juicio, uno de los inconvenientes es el escaso número de cirugías tuteladas, especialmente en la prostatectomía radical.

Por otro lado los "fellowship" muy populares en el medio norteamericano y en auge en los centros de excelencia europeos, son programas de inmersión en una técnica concreta que permiten al urólogo una dedicación exclusiva, no interferida por la actividad asistencial, durante un periodo que oscila entre 6 y 12 meses. Existen datos de la eficiencia de dichos programas, así Pasandoro y cols, contactaron con 14 becarios un año después de acabado su programa de formación y 12 de ellos realizaban con 
Tabla 4. Comparativa de las intervenciones realizadas por urólogos recién terminado su programa de formación tutelado

\begin{tabular}{|c|c|c|c|c|c|c|c|}
\hline & Serie Global ${ }^{18}$ & Residente $1^{2}$ & Residente $^{2}$ & Fellow & Fabricio et al. ${ }^{13}$ & Skrekas et al. ${ }^{14}$ & Raventós et al. ${ }^{15}$ \\
\hline $\mathrm{N}$ & 1300 & 50 & 50 & 30 & 20 & 16 & 21 \\
\hline Tiempo (min) & 153 & $176(110-285)$ & $173(110-270)$ & 147 (80-190) & $313(180-602)$ & $386(280-480)$ & 198 \\
\hline LP & 46,8 & $26 \%$ & $24 \%$ & $66,67 \%$ & $\mathrm{Nd}$ & $37,5 \%$ & $\mathrm{Nd}$ \\
\hline Transfusión & $0,9 \%$ & $4 \%$ & 0 & 0 & 0 & 0 & $\mathrm{Nd}$ \\
\hline Complicaciones & $102 / 1300$ & $\begin{array}{c}2 \text { lesiones } \\
\text { de recto }\end{array}$ & 0 & 0 & & $\begin{array}{c}\text { Insuficiencia } \\
\text { de la anastomosis }\end{array}$ & $\mathrm{Nd}$ \\
\hline Retirada sonda & 6,2 & $7,6(4-20)$ & $6,4(4-20)$ & $(4-7)$ & $9(6-22)$ & $12,7(6-30)$ & \\
\hline Márgenes Qx & & & & $6,66 \%$ & $30 \%$ & $37,5 \%$ & 0 \\
\hline pT2 & $9,8 \%$ & $14,3 \%$ & $11,5 \%$ & $3,84 \%$ & & & \\
\hline pT3 & $34,3 \%$ & $38 \%$ & $29 \%$ & $25 \%$ & & & \\
\hline
\end{tabular}

LP: Linfadenectomía prostática. Márgenes Qx: quirúrgicos.

asiduidad la técnica aprendida, refiriendo buenos resultados y una tasa de reconversión menor del $2 \%^{10}$. Del mismo modo, Colegrove y cols precisaron que los becarios de sus centros realizaban un mínimo de 25 casos anuales ${ }^{11}$.

Como ya hemos precisado el Centro Internacional de Formación laparoscópica de Leipzig ofrece una Beca o "fellowship" a tiempo completo (6-8 meses). Gracias a la amplia casuística, durante dicho periodo conviven "observers" noveles, el propio alumno visitó la clínica de Leipzig durante un total de 6 semanas durante los años previos a la beca; asî como expertos en laparoscopia que acuden a observar las modificaciones de la técnica intrafascial ${ }^{6}$. Del mismo modo asisten urólogos cuya estancia oscila entre una y ocho semanas para la realización de las tutorías formativas intermitentes.

Desde el año 2004 el centro recibe 2 becarios anuales y en el 2005 se estandarizó en programa de entrenamiento modular ${ }^{12}$. Todos los becarios cumplimentaron el programa, alcanzando el módulo último que incluye las modificaciones para la conservación de bandeletas vasculonerviosas. Todos practican de manera regular la técnica EERPE en sus centros de trabajo. Sin embargo, quedan pendientes conocer los datos de las cirugías realizadas por dichos becarios en sus centros hospitalarios, es de esperar cuanto menos un aumento en los tiempos quirúrgicos y en las complicaciones menores. No obstante adelantamos que los datos disponibles auguran que la técnica es reproducible y que el entrenamiento modulado tutorizado optimiza el aprendizaje del mismo, reduciendo a su mínima expresión la tasa de complicaciones.
Si revisamos otros programas de formación tutelados, observamos que mientras el becario del presente trabajo necesitó de 52 casos donde intervino realizando alguno de los módulos, los grupos de Virginia ${ }^{13} \mathrm{y}$ Amsterdan ${ }^{14}$ consideran que tras $12-16$ casos de ayudantía los resultados son equiparables a los del propio tutor. A nuestro juicio sin una incursión progresiva en la técnica, alcanzar la solvencia quirúrgica con tan corta casuística es extremadamente complicado. Probablemente el sesgo que supone que en dichos trabajos el alumno tenga más de 150 cirugías laparoscópicas renales como experiencia previa, justifiquen el rápido aprendizaje. Es obvio que la experiencia de los urólogos analizados en sendos trabajos dista de la de los residentes españoles, por lo que la evidencia obtenida no es aplicable en nuestro medio. No obstante al analizar pormenorizadamente los datos, objetivamos que en el trabajo de Fabricio et al. la tasa de márgenes positivos se incrementa de manera relevante aunque no significativa cuando el alumno realiza la cirugía como responsable ${ }^{13}$, pasando del 9 al $22 \%$.

En nuestro medio, Raventos y cols. describieron la curva de aprendizaje tutorizada de 5 alumnos con 180 prostatectomías radicales. En dicho trabajo cada uno de los alumnos realizó al menos 2 cirugías sin el tutor presente ${ }^{15}$.

El grupo de Leipzig publicó la curva de aprendizaje de los dos primeros residentes con los que se desarrolló el programa de formación, dichos residentes requirieron 43 y 38 casos para ser considerados competentes $^{2}$. Los resultados de las prostatectomías radicales realizadas tras la cumplimentación del entrenamiento fueron comparados con los de la serie histórica del tutor, sin objetivar diferencias ${ }^{2}$. 
La curva de aprendizaje que se describe en el presente artículo requirió de 52 casos. Probablemente estas diferencias las expliquen el hecho de que los anteriores residentes actuaron como primeros ayudantes durante la curva de aprendizaje del tutor durante dos años, además tenían cierta experiencia en procedimientos laparoscópicos de menor envergadura como la reparación de hernias por vía extraperitonal y varicocelectomías ${ }^{2}$.

En la Tabla 4 comparamos los primeros casos de los residentes del grupo de Leipzig, los del último "fellow" en dicho centro, y los de los grupos de Virginia, Ámsterdam y Español. Observamos que los tiempos quirúrgicos en todos los grupos son aceptables, aunque notablemente menores en el grupo alemán incluso incluyendo la linfadenectomía pélvica en una alta proporción de pacientes y la corrección de hernias inguinales con malla según la técnica descrita previamente ${ }^{16}$.

En líneas generales las diferencias entre estos grupos son anecdóticas, sin embargo Pena González et al. ${ }^{17}$ con 50 pacientes y sin un aprendizaje claramente tutorizado refieren 11 reconversiones, una revisión quirúrgica postoperatoria por sangrado, 3 fugas de orina relevantes por la anastomosis que retrasan el alta, una anastomosis incompetente, un atrapamiento del meato ureteral durante la sutura ${ }^{17}$, de modo que sin un aprendizaje tutorizado, y progresivo los tiempos quirúrgicos, la tasa de reconversiones de transfusiones y de complicaciones aumenta de manera relevante.

Por consiguiente, esta revisión confirma la bondad del aprendizaje tutelado progresivo. La reproducibilidad de la EERPE es la característica princeps que se desprende de este trabajo. A nuestro juicio quedan relegadas a la historia las fases de reconversión programada que los laparoscopistas pioneros propugnaban para iniciarse en esta técnica, de hecho, lo deseable sería que la próxima generación de urólogos que comienza ahora su formación en los centros terciarios cumplimentaran su curva de aprendizaje tutelada durante su periodo de residencia, de modo que cuando se trasladen a centros secundarios puedan desarrollar los procedimientos sin reducir excesivamente la eficiencia de la técnica.

\section{CONCLUSIÓN}

Con el modelo de formación tutelado por módulos de dificultad creciente, los becarios alcanzan en el centro de formación el nivel necesario para la realización de la técnica sin un ayudante experto, en unos tiempos quirúrgicos y unas tasas de complicaciones óptimas. Este trabajo confirma la eficiencia del modelo de formación tutelado por módulos de dificultad creciente diseñado por el grupo alemán (EERPE). Siendo conscientes de las limitaciones que supone la menor casuística en nuestros centros, trasladar a nuestro hospital universitario dicho programa de formación es el nuevo reto que nos debiera ocupar.

\section{REFERENCIAS}

1. Cansino Alcaide JR, Álvarez Maestro M, Castillo Cabrera PM, Martínez-Pineiro Lorenzo L Tabernero Prieto A, De la Pena Barthel JJ. Prostatectomía radical laparoscópica. Revisión de la literatura. Nuestra experiencia. Actas Urol Esp. 2006;30(5):517-530.

2. Stolzenburg JU, Rabenalt R, Do M, Horn LC and Liatsikos E. Modular training for residents with no prior experience with open pelvic surgery in endoscopic extraperitoneal radical prostatectomy. Eur Urol. 2006;49(3):491-498.

3. Ganzer R, Rabenalt R, Truss MC, Papadoukakis S, Do M, Blana A, Straub M et al. Evaluation of complications in endoscopic extraperitoneal radical prostatectomy in a modular training programme: a multicentre experience. World J Urol. 2008;26(6): 587-593.

4. Stolzenburg JU, Rabenalt R, Do M, Jimenez Cruz F, Liatsikos E. Prostatectomía radical extraperitoneal laparoscópica: evolución en el tiempo y resultados actualizados. Actas Urol Esp. 2006;30(6):556-566.

5. Stolzenburg JU, Bynens B, Do M, Rabenalt R, Katsakiori PF, Liatsikos E Salvage laparoscopic extraperitoneal radical prostatectomy after failed highintensity focused ultrasound and radiotherapy for localized prostate cancer. Urology. 2007;70(5):956-960.

6. Stolzenburg JU, Rabenalt R, Do M, Schwalenberg T, Winkler M, Dietel A Liatsikos E. Intrafascial Nerve-Sparing Endoscopic Extraperitoneal Radical Prostatectomy. Eur Urol. 2008;53(5): 931-940.

7. Stolzenburg $\mathrm{J}$ and Truss M. Technique of laparoscopic (endoscopic) radica prostatectomy. BJU Int. 2003;91(8):749-757.

8. Bollens R, Sandhu S, Roumeguere T, Quackels T, Schulman C. Laparoscopic radical prostatectomy: the learning curve. Curr Opin Urol. 2005;15(2):79-82.

9. Barnes RW, Lang NP and Whiteside MF. Halstedian technique revisited Innovations in teaching surgical skills. Ann Surg. 1989;210(1):118-121.

10. Pansadoro A, Curto F, Mugnier C, Hoepffner JL, Gaston R and Piechaud T. Teaching laparoscopy: the new challenge. BJU Int. 2007;99(4):726-727.

11. Colegrove PM, Winfield HN, Donovan JF, Jr. and See WA. Laparoscopic practice patterns among North American urologists 5 years after formal training. J Urol. 1999;161(3):881-886.

12. Stolzenburg JU, Schwaibold H, Bhanot SM, Rabenalt R, Do M, Truss M et al. Modular surgical training for endoscopic extraperitoneal radical prostatectomy. BJU Int. 2005;96(7): 1022-1027

13. Fabrizio MD, Tuerk I, Schellhammer PF. Laparoscopic radical prostatectomy: decreasing the learning curve using a mentor initiated approach. J Urol. 2003;169(6):2063-2065.

14. Skrekas T, Mochtar CA, Lagerveld BW, de Reijke TM, van Velthoven RF, Peltier A et al.: Mentor-initiated approach in laparoscopic radical prostatectomy. J Endourol. 2006;20(10): 831-835

15. Raventos Busquets CX, Cecchini Rosell L, Trilla Herrera E, Planas Morin J, Orsola de los Santos A, Morote Robles J: Prostatectomía radical laparoscópica: la curva de aprendizaje. Actas Urol Esp. 2007;31(3):205-10.

16. Stolzenburg J, Rabenalt R, Dietel A, Do M, Dorschner W, Truss M: Hernia Repair during endoscopic (laparoscopic) radical prostatectomy. J Laparoendosc Adv Surg Tech A. 2003 Feb; 13(1):27-31

17. Pena Gonzalez JA, Gonzalez Sala JL, Prera Vilaseca A, Abad Gairin C, Graells Batet A, Descalzo Abad MC. Prostatectomía radical laparoscópica extraperitoneal. Primeros 50 casos en un centro comarcal. Análisis de la curva de aprendizaje. Actas Urol Esp. 2007;31(7):732-737.

18. Stolzenburg JU, Rabenalt R, Do M, Truss MC, Burchardt M, Herrmann TR et al. Endoscopic extraperitoneal radical prostatectomy: the University of Leipzig experience of 1,300 cases. World J Urol. 25: 45-51, 2007.

Correspondencia autor: Dr. Miguel Ramírez Backhaus

Servicio de Urología. Hospital Universitario La Fe.

Avenida Campanar, 21 - 46009 Valencia. Tel.: 963862700

E-mai autor: ramirezbackhaus@yahoo.es

Información artículo: Original - Laparoscopia

Trabajo recibido: noviembre 2008

Trabajo aceptado: diciembre 2008 